\title{
Modal Sensitivity Based Sensor Placement for Damage Identification Under Sparsity Constraint
}

\author{
Bartlomiej Blachowski ${ }^{1 *}$ \\ 1 Institute of Fundamental Technological Research, \\ Polish Academy of Sciences \\ Pawinskiego 5b, 02-106 Warsaw, Poland \\ *Corresponding author, e-mail: bblach@ippt.pan.pl
}

Received: 15 February 2019, Accepted: 13 March 2019, Published online: 26 March 2019

\begin{abstract}
The present study deals with a comprehensive approach for damage identification of spatial truss structures. The novelty of the proposed approach consists of a three-level analysis. First, sensitivity of assumed modal characteristics is calculated. Second, natural frequency sensitivity is used to determine hardly identifiable structural parameters and mode shape sensitivity is applied to select damage-sensitive locations of sensors. Third, two sparsity constrained optimization algorithms are tested towards efficient identification of applied damage scenarios. These two algorithms are based on $\ell_{1}$-norm minimization and non-negative least square (NNLS) solution.

Performances of both proposed algorithms have been compared in two realistic case studies: the first one concerned a threedimensional truss girder with 61 structural parameters and the second one was devoted to an upper-deck arch bridge composed of 416 steel members.
\end{abstract}

\section{Keywords}

sensor placement, damage identification, $\ell_{1}$-norm minimization, sparsity constrained optimization

\section{Introduction}

Optimal sensor placement (OSP) and structural damage detection have been topics of intensive research for over thirty years. The motivation behind that research is the fact that many civil structures reach the end of their service life and structural health monitoring becomes the critical aspect in assuring their safety and reliability.

One of the first papers devoted to optimization of sensor placement for parameter estimation in structural systems subjected to an earthquake excitation has been published by Shah and Udwadia [1]. A year later the idea of damage detection from changes in natural frequencies has been introduced by Cawley and Adams [2].

After these pioneering works a significant increase of interest in that field of research took place and in particular in the 90 s of the last century a number of important papers were published. The Effective Independence (EI) technique has been introduced by Kammer [3] for efficient placement of accelerometers on large truss structures. Parametric identification of trusses using static strain measurements has been investigated by Liu and Chian [4]. Their conclusion was that even when strains in all elements are measured it can be impossible to identify structural parameters in a complex truss structure using only a single static loading pattern.

It was one of the reasons why researchers oriented their interests towards dynamic excitation based techniques. Among many papers from that period an interesting study has been published by Cobb and Liebst [5]. They pointed out a critical aspect of sensor placement and its influence on the ability to identify damaged elements in three-dimensional trusses.

Contrary to the studies by either Cobb and Liebst, or Shah and Udwadia, which used modal and response sensitivity matrices for parameter estimation, an interesting novel approach has been proposed by Beck and Katafygiotis [6]. In their two-part paper they introduced the Bayesian inference concept to parametric estimation of multi degree of freedom systems and concluded that 
improperly located sensors can result in incorrect estimation of stiffness parameters. Further exploration of the Bayesian approach and information theory and their application to the sensor placement problem has been done by Papadimitriou [7]. He used the concept of information entropy (IE) and proposed a computationally efficient sequential sensor placement technique giving results similar to those yielded by a genetic algorithm.

In 2009 an excellent overview of existing techniques for optimal sensor placement was published by Barthorpe and Worden [8]. Recently, Zhou et al. [9] have proposed a software architecture implementing four approaches for OSP, namely: mode shape difference, Effective Independence, information entropy and modal energy. Besides sensor placement optimization, an important aspect of any modal characteristic based SHM system is the solver used for parametric estimation.

Early works from the 90 -ties suggested $\ell_{2}$-norm minimization, an example being the distinguished book by Friswell and Mottershead [10]. However, researchers are currently reporting the $\ell_{1}$-norm minimization technique as a most accurate in structural parameter identification (Hernandez [11]). Additionally, SHM techniques can be implemented as off-line or on-line algorithms. Among the latter it is worth mentioning the works by Ebrahimian et al. [12] utilizing the Extended Kalman Filter (EKF) approach or by Grinsberg et al. [13] utilizing sparsity constrained EKF.

Currently used methods for damage identification can be classified as either time or modal domain based ones. Representative examples belonging to the first group are the work by An et al. [14], where curvature between two neighboring measurement points has been used to identify damage in a simple beam structure, by Pnevmatikos et al. [15], where a wavelet based technique has been used to localize damage in a planar frame structure, or by Blachowski et al. [16], in which changes in axial strain accelerations have been applied to damage localization in a spatial truss structure. Methods from the second group have been investigated by An et al. [17], who utilized QR decomposition to detect changes in the flexibility matrix based on measured modes, or by Blachowski et al. [18] for detection of damage in a bolted lap connection. Damage in flanged connections of tall steel towers was the topic of the paper by Blachowski and Gutkowski [19]. Recently, a time domain technique for simultaneous sensor placement and damage identification in truss structures has been proposed by Blachowski et al. [20].
Damage identification using three different objective functions based on residuals obtained from static and modal measurements has been proposed by Kourehli [21]. He applied simulated annealing to verify his method on a four-story steel frame proposed as IASC-ASCE structural health monitoring benchmark. Bayer et al. [22] proposed damage localization using a criterion defined as the product of selected mode flexibility and the modal curvature. Experimental setup consisted of 3-meter long simply supported beam with rectangular hollow section. Six mode shape have been used to properly localize damage within the beam. An efficient damage index for multiple damage identification using the concepts of frequency response function (FRF) and strain energy has been proposed by Bagherahmadi and Seyedpoor [23].

At the end of the overview of damage identification methods one has to mention the problems with the deployment of Structural Health Monitoring systems for damage identification. An interesting alternative for acceleration or strain measurements for evaluation of dynamic structural response has been proposed by Beben [24]. He applied an interferometric radar for dynamic testing of various bridges. However, as he concluded the method is suitable for small-to-medium scale bridges.

As one can conclude from the above literature overview, there is continuous progress towards efficient SHM systems for damage identification. However, there are still relatively few comprehensive studies of the design of such a SHM system taking into account all aspects of the issue, namely: sensor placement, sensitivity to structural parameter changes caused by damage and finally the frequency range of excitation. This work is a preliminary attempt to fill this gap.

The present study proposes a comprehensive methodology for damage quantification in large truss structures. The methodology is composed of modal sensitivity based sensor placement together with sparsity constrained damage identification. For the purpose of sparsity constrained optimization two solvers will be considered. The first one is based on the non-negative least squares (NNLS) algorithm and the second one utilizes $\ell_{1}$-norm minimization subject to equality constraints as implemented in the 11-magic toolbox. Effectiveness of the proposed methodology will be verified in two case studies. The first one employs a laboratory-scale truss structure with 61 structural elements and the second one deals with real-scale arch bridge used in Japan as a benchmark for verification of seismic design quality. 


\section{Proposed methodology for damage-sensitive} sensor placement and sparsity constrained damage identification

Before detailed description of the proposed methodology three important aspects of any Structural Health Monitoring system devoted to damage identification will be briefly presented. They are: a) damage type and location, b) number, type and distribution of allowable sensors and finally c) source of excitation (Fig. 1). Below a short description of the above aspects is given:

\section{a) Damage type and location}

First of all, when designing a successful SHM system one should be aware that there is no universal SHM system able to detect all possible damage scenarios. The main difficulty is to properly characterize which of all possible scenarios can occur first and then parametrize the structure under investigation in such a way that this damage scenario is included in the given parametrization.

b) Number, type and distribution of sensors

The second important aspect is related to acquired measurement data. It is well known that poorly distributed sensors are not able to capture crucial characteristics of the structure. Therefore, it is critical to consider this aspect before installing a monitoring system on the investigated structure.

c) Source of excitation

Similarly to the sensor placement aspect, location and frequency range of excitation can have significant influence on the performance of any SHM system.

Taking into account the above issues the present work proposes a comprehensive algorithm for solving damage identification by means of low-frequency vibrations. Outline of the proposed methodology is presented in a stepby-step manner as Algorithm (Table 1) or in a graphical form as flowchart (Fig. 2).

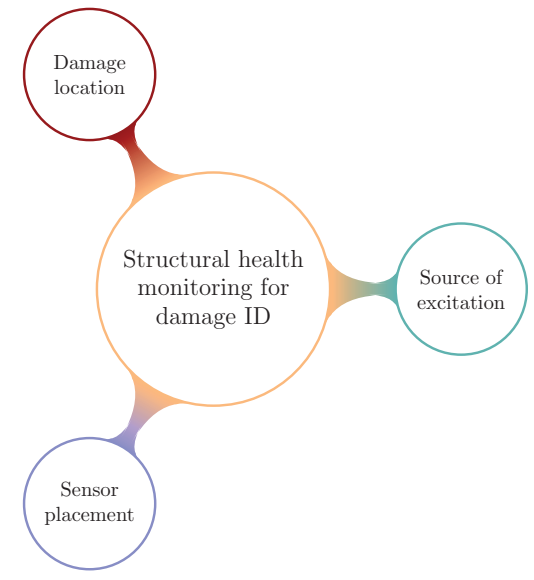

Fig. 1 Three critical aspects of vibration-based SHM
Table 1 Sensor placement for sparsity constrained damage identification

\begin{tabular}{l}
\hline Algorithm: \\
\hline 1. Choose a proper parametrization for the expected type of damage \\
(Eq. (2)) \\
2. Calculate frequency and mode shape sensitivity matrices for the \\
modes within the excitation range (Eq. (4) and (5)) \\
3. Create a rating of damage-sensitive degrees of freedom for \\
potential sensor placement (Eq. (6)) \\
4. Select uncorrelated locations for optimal sensor placement (Eq. (7)) \\
5. Identify damage using sparsity constrained optimization \\
algorithms (Eq. (8)) \\
6. Evaluate the overall damage identification system \\
7. If the required criteria are not satisfied, return to 1. and depending \\
on the available resources perform one of the following: \\
(a) Change the assumed parameterization \\
(b) Modify the source of excitation to increase the number of \\
modes in the structural response
\end{tabular}

(c) Increase the number of sensors

otherwise save the optimal sensor placement and finish the algorithm

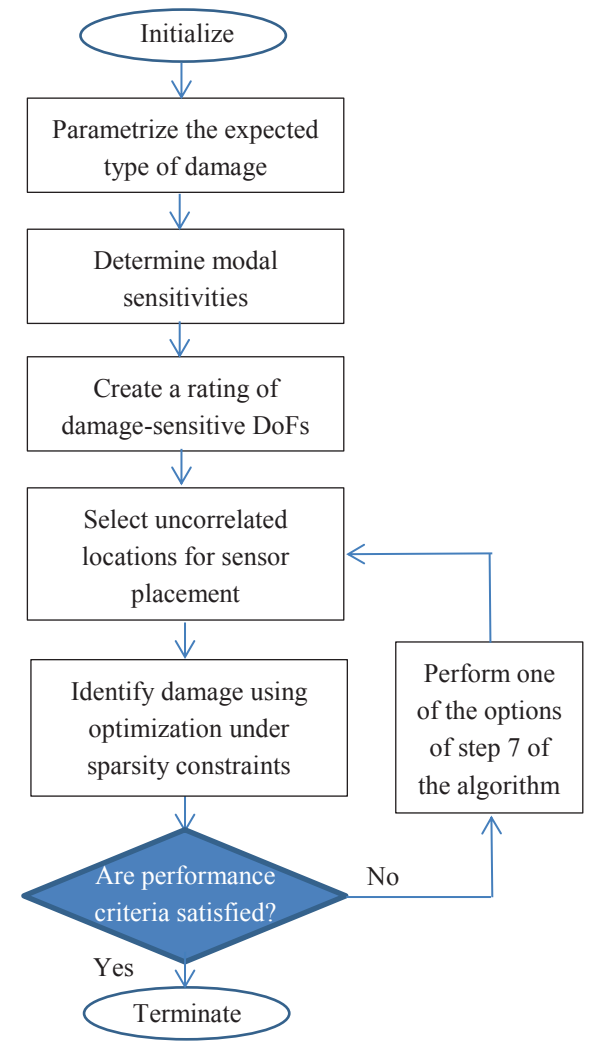

Fig. 2 Flowchart of the proposed algorithm

The subsequent sections of this paper are devoted to these three aspects. The next section deals with a parametrization suitable for stiffness degradation in structural members of trusses. Section 2.2 will present a damage sensitive sensor placement methodology. And finally, Section 2.3 will describe a sparsity constrained damage 
identification method based on knowledge of a set of natural frequencies and mode shapes. Wrong excitation will not allow proper identification of the assumed modes and can cause the whole SHM system to not work correctly.

\subsection{Damage parametrization in modal sensitivity based methods}

Measurement of low frequency vibrations of an engineering structure is the most frequently utilized technology in designing a health monitoring system. This is because such vibrations provide a global characteristic of the structure's condition. Two competitive approaches for damage identification are distinguished here: the first one is performed in time-domain and is based on dynamic response modification and the second one in modal-domain uses changes in natural frequencies and mode shapes. Although, the latter approach is criticized for its lower sensitivity as compared to the former, its unquestionable advantage is an easier formulation for random excitation, which is frequently ambient and unknown. Therefore, in the case of large scale civil structures modal parameters based approaches are very often the only option.

The derivation of the proposed methodology for damage identification is initiated by recalling the fundamental equation involved in modal parameter extraction. The undamped eigenvalue problem of a multi-degree of freedom structure is described by the well-known set of algebraic equations

$$
\left(\mathbf{K}-\omega_{k}^{2} \mathbf{M}\right) \phi_{k}=0, \quad k=1,2, \ldots n_{m},
$$

where $\mathbf{K}$ and $\mathbf{M}$ are the stiffness and mass matrix, respectively, $\omega_{k}$ and $\phi_{k}$ denote the $k$-th natural frequency and mode shape, respectively, finally $n_{m}$ is the number of modes under investigation.

The above equation serves as a tool to predict modal pairs (natural frequency and corresponding mode) of the structure, for which structural parameters might differ from the nominal ones. For that reason, at this stage it is necessary to introduce the parametrization describing structural modifications caused by damage. Additionally, it is reasonable to assume that in a deteriorating structure only stiffness parameters will change and mass properties, identified at an early stage, will not change during the service life of the structure. Taking the above assumptions into account the parametrization used in this study takes the following form

$$
\mathbf{K}=\mathbf{K}_{0}-\sum_{j=1}^{n_{p}} \theta_{j} \mathbf{K}_{j},
$$

where $\mathbf{K}_{0}$ is the nominal stiffness matrix (obtained by calibration of the initial FEM using experimental data from a healthy structure), $\mathbf{K}_{j}$ is the stiffness matrix corresponding to modification of the $j$-th stiffness parameter. In this study such a parameter will be related to the stiffness of an individual truss member, but generally it can describe stiffnesses of a set of truss elements constituting the $j$-th substructure.

Having parametrized the structure under investigation we can express the modal parameter of the damaged structure in terms of the healthy one using Taylor expansion

$b_{d}=b_{0}-\left.\frac{\partial b}{\partial \theta_{j}}\right|_{b=b_{0}} \Delta \theta_{j}+O\left(\Delta \theta_{j}^{2}\right)$.

In Eq. (3) $b_{d}$ denotes the modal parameter (a natural frequency or a single component of the mode shape vector) of the damaged structure, $b_{0}$ is the corresponding modal parameter of the healthy structure, and $\partial b / \partial \theta_{j}$ denotes the derivative of this modal parameter with respect to the $j$-th structural parameter. In the case of many modal and structural parameters the above equation takes vector form and corresponding derivative is a whole matrix called sensitivity. A detailed derivation of this matrix will be the topic of the subsequent section.

\subsection{Damage-sensitive sensor placement methodology}

The derivative of modal Eq. (1) with respect to the structural parameter takes the following form

$\frac{\partial}{\partial \theta_{j}}\left(\mathbf{K}-\omega_{k}^{2} \mathbf{M}\right) \phi_{k}+\left(\mathbf{K}-\omega_{k}^{2} \mathbf{M}\right) \frac{\partial}{\partial \theta_{j}} \boldsymbol{\phi}_{k}=\mathbf{0}$.

Multiplying on the left by $\phi_{k}^{\mathrm{T}}$ and using the undamped eigenvalue solution the derivative can be written as

$\phi_{k}^{\mathrm{T}} \frac{\partial}{\partial \theta_{j}}\left(\mathbf{K}-\omega_{k}^{2} \mathbf{M}\right) \phi_{k}=\mathbf{0}$,

or more explicitly

$\phi_{k}^{\mathrm{T}}\left(\frac{\partial \mathbf{K}}{\partial \theta_{j}}-2 \omega_{k} \frac{\partial \omega_{k}}{\partial \theta_{j}} \mathbf{M}-\omega_{k}^{2} \frac{\partial \mathbf{M}}{\partial \theta_{j}}\right) \phi_{k}=\mathbf{0}$.

Assuming that there is no change in the mass matrix and utilizing the orthogonal property of the eigenvalue problem, $\phi_{k}^{\mathrm{T}} \mathbf{M} \phi_{l}=0$ for $k \neq l$, with mass-normalized eigenvectors $\phi_{k}^{\mathrm{T}} \mathbf{M} \phi_{k}$ the above equation can be further simplified to

$$
\frac{\partial \omega_{k}}{\partial \theta_{j}}=\phi_{k}^{\mathrm{T}} \frac{\partial \mathbf{K}}{\partial \theta_{j}} \boldsymbol{\phi}_{k}
$$


Determining the derivative of the $k$-th natural frequency with respect to the $j$-th structural parameter allows one to create the whole matrix, which will be referred to here as the sensitivity matrix ${ }^{\omega} \mathbf{S}$.

Having determined the sensitivity of a natural frequency one can proceed to obtain the derivative of the mode shape with respect to structural parameters. For that purpose we return to the derivative of the eigenvalue problem (Eq. (1)) and omit the left multiplication step. Next, using the Nelson technique [25] we can calculate the mode shape sensitivity as follows:

First, we define the $\mathbf{f}_{k}$ vector

$\mathbf{f}_{k}=\left(\frac{\partial \mathbf{K}}{\partial \theta_{j}}-2 \omega_{k} \frac{\partial \omega_{k}}{\partial \theta_{j}} \mathbf{M}\right) \phi_{k}$.

Next, we find the index of the maximum component of the absolute value of the $k$-th mode shape. Using this index, we make the following partitioning of the dynamic stiffness matrix

$$
\left[\begin{array}{c}
\mathbf{v}_{1} \\
v_{0} \\
\mathbf{v}_{2}
\end{array}\right]_{k}=\left[\begin{array}{ccc}
\left(\mathbf{K}-\omega_{k}^{2} \mathbf{M}\right)_{11} & 0 & \left(\mathbf{K}-\omega_{k}^{2} \mathbf{M}\right)_{12} \\
0 & 1 & 0 \\
\left(\mathbf{K}-\omega_{k}^{2} \mathbf{M}\right)_{21} & 0 & \left(\mathbf{K}-\omega_{k}^{2} \mathbf{M}\right)_{22}
\end{array}\right]^{-1}\left[\begin{array}{c}
\mathbf{f}_{1} \\
0 \\
\mathbf{f}_{2}
\end{array}\right]_{k}
$$

Finally, mode shape sensitivity denoted here as ${ }^{\phi} \mathbf{S}$ can be determined using the following formula

$$
\frac{\partial \boldsymbol{\phi}_{k}}{\partial \theta_{j}}=\mathbf{v}_{k}-\boldsymbol{\phi}_{k}^{\mathrm{T}} \mathbf{M} \mathbf{v}_{k} \boldsymbol{\phi}_{k} .
$$

It should be noted here that the mode shape sensitivity matrix is a three dimensional object, which is presented schematically in Fig. 3. Such an object is described by three indices ${ }^{\phi} s_{i, j}^{(k)}$. The meaning of the individual indices is as follows: the first index $i$ represents degrees of freedom, the second index $j$ is associated with structural parameters, and finally the third index $k$ corresponds to subsequent modes of the structure.

For the above mode shape sensitivity matrix we can define cumulative metrics for the $i$-th degree of freedom

${ }^{\phi} d_{i}=\sum_{j=1}^{n_{p}} \sum_{k=1}^{n_{m}}\left|{ }^{\phi} S_{i, j}^{(k)}\right|$.

Such metrics give us information about the sensitivity of an individual DoF to changes in structural parameters. The DoFs with the highest sensitivity are good candidates for sensor locations. The method is very simple and efficient, but it has one drawback. The DoFs selected in this way are often sensitive to the same structural parameters,

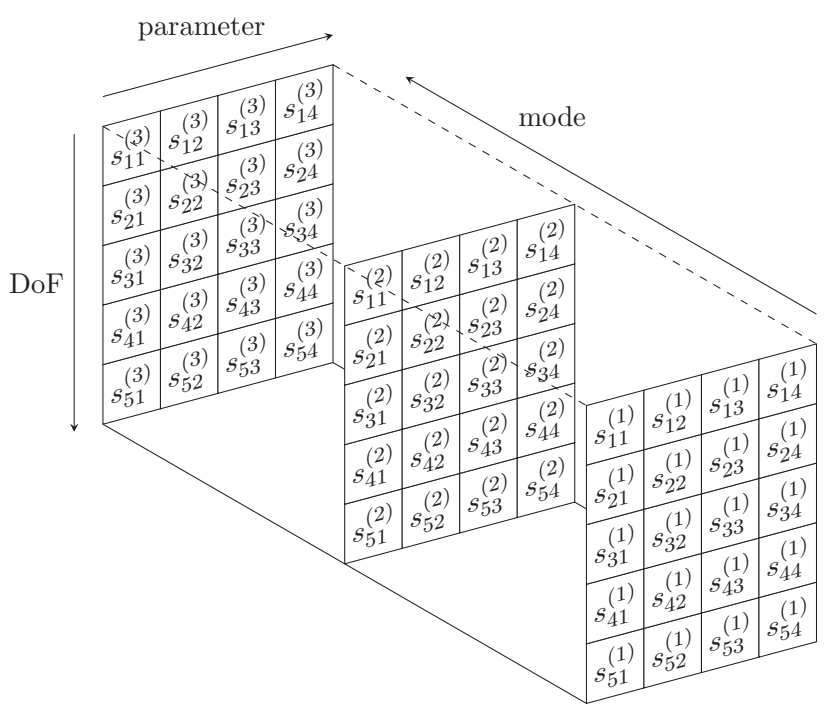

Fig. 3 Mode shape sensitivity matrix

so one should provide additional information about the mutual correlation of the corresponding rows of the sensitivity matrix. If these rows are highly correlated, another location with smaller metrics should be chosen. Mathematically, we can express this issue using the following co-linearity matrix

${ }^{\phi} \boldsymbol{\psi}=\frac{1}{n_{m}} \sum_{k=1}^{n_{m}} \overline{\mathbf{S}}^{(k) \phi} \overline{\mathbf{S}}^{(k) \mathrm{T}}$,

where ${ }^{\phi} \overline{\mathbf{S}}^{(k)}$ denotes the row normalized sensitivity matrix for the $k$-th mode shape.

Application of the presented modal sensitivity analysis to both sensor placement and damage identification will be the topic of subsequent sections.

\subsection{Sparsity constrained optimization for damage identification}

While the previous section introduced the sensitivity matrix for sensor placement purposes, the present section utilizes this matrix for damage identification. Since the most effective method for damage identification should be able to detect damage-induced parametric modification as early as possible in this study it is assumed that a change in structural parameters can be determined using the first order term of the Taylor expansion of modal characteristics. The corresponding set of equations is usually expressed in the following form

$\Delta \mathbf{b}=\mathbf{S} \Delta \boldsymbol{\theta}$,

where $\Delta \mathbf{b}$ represents the difference between measured and calculated modal characteristics, $\mathbf{S}=\left[{ }^{\omega} \mathbf{S},{ }^{\phi} \mathbf{S}^{(1)},{ }^{\phi} \mathbf{S}^{(2)}, \ldots\right.$, $\left.\mathbf{S}^{\left(n_{m}\right)}\right]^{\mathrm{T}}$ is the overall sensitivity matrix composed of two 
matrices representing natural frequency and mode shape sensitivities, respectively, and finally $\Delta \boldsymbol{\theta}$ is the difference between nominal and modified structural parameters.

Generally, the approach for finding the solution to the above set of equations depends on dimensions of the sensitivity matrix $\mathbf{S} \in \mathbb{R}^{n_{d} \times n_{p}}$, where $n_{p}$ is the number of available measurement data and $n_{p}$ is the number of unknown parameters. In the case of an over-determined set of equations ie. $n_{d} \geq n_{p}$ most frequently a solution with the minimal $\ell_{2}$-norm of the residual vector $\|\mathbf{S} \Delta \boldsymbol{\theta}-\Delta \mathbf{b}\|_{2}$ is satisfactory. However, in the low frequency based damage identification the situation is rather different and we have fewer data than parameters to identify, ie. $n_{d}<n_{p}$. In such a situation infinitely many solutions can exist and one has to specify additional constraints to find an appropriate solution.

In the case of an under-determined set of equations the simplest solution is called basis solution and can be obtained in MATLAB by applying the matrix left division operator " $\backslash "$. As a result we get a solution in the form of a vector with $r$ non-zero elements, where $r$ is the rank of the sensitivity matrix. Another option is the minimum $\ell_{2}$-norm solution, but in the under-determined case the norm for the unknown parameters is minimized and such a solution can be obtained using a pseudo-inverse of the sensitivity matrix pinv(S). Unfortunately, neither matrix left division nor pseudo-inverse can provide a good estimate of the structural parameters in general case of a damage scenario.

To overcome the above issue nowadays researchers frequently use approaches based on regularization, where a certain parameter $\lambda$ is used to make a trade-off between two norms: the first one for residuals and the second one for uncertain parameters

$$
\min _{\Delta \theta}\|\mathbf{S} \Delta \theta-\Delta \mathbf{b}\|_{2}^{2}+\lambda\|\Delta \theta\|_{2}^{2}
$$

The disadvantage of the above formulation is the lack of a general method for appropriate selection of the regularization parameter $\lambda[26]$.

Recently, a new idea has appeared, on the basis of which the solution can be obtained assuming sparsity of the vector of unknown parameters. In this formulation we solve the following optimization problem:

$\min _{\Delta \boldsymbol{\theta}}\|\Delta \boldsymbol{\theta}\|_{1}$ subject to $\mathbf{S} \Delta \boldsymbol{\theta}-\Delta \mathbf{b}=\mathbf{0}$.

The sparsity is enforced by the $\ell_{1}$-norm, which is nothing else than sum of absolute values of the individual components of $\Delta \boldsymbol{\theta}$. An implementation of this optimization problem can be found in the 11-magic toolbox authored by Candes and Romberg. As it will be demonstrated in the section devoted to numerical examples, the above formulation can also provide large estimation error of the structural parameters.

Additionally, the most accurate parameter estimation has been obtained using the least squares solution with non-negativity constraints. The mathematical formulation of the optimization problem is as follows:

$\min _{\Delta \boldsymbol{\theta}}\|\mathbf{S} \Delta \boldsymbol{\theta}-\Delta \mathbf{b}\|_{2}^{2}$ subject to $\Delta \boldsymbol{\theta}>\mathbf{0}$.

The non-negative least squares (NNLS) algorithm was published originally by Lawson and Hanson. This algorithm has been implemented in MATLAB and can be invoke using lsqnonneg command.

\section{Numerical examples}

The theoretical considerations presented in the previous sections will be illustrated with two case studies. The first one is a laboratory-scale three-dimensional truss structure shown in Fig. 4. and the second one is a full-scale steel arch bridge used as a benchmark problem for seismic design (Fig. 14).

\subsection{MERO truss - 61 member structure}

The case study presented in this subsection is based on a commercially available exhibit system, known as MERO M12 [27]. The structure spans $3.5 \mathrm{~m}$ and consists of 61 steel members with circular hollow sections. The dimensions of the cross section are: $22 \mathrm{~mm}$ in diameter, $1 \mathrm{~mm}$ of wall thickness. Young's modulus $E$, Poisson ratio $v$ and mass density $\rho$ are equal to $205 \mathrm{GPa}, 0.3$ and $7850 \mathrm{~kg} / \mathrm{m}^{3}$, respectively.

The structure is simply supported with one end fixed and the other resting on a roller, which allows movement in the longitudinal direction. Additionally, the structure is assembled using screws located at the end of each tubular member. This in turn causes transfer of bending moments between neighboring tubes.

This fact was reflected in the numerical model by assuming that the whole structure behaves as a spatial frame. The first four mode shapes of the structure and corresponding natural frequencies are presented in Fig. 5.

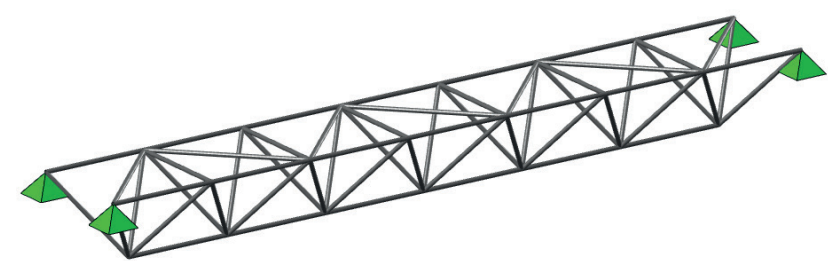

Fig. 4 MERO truss $-3 \mathrm{D}$ view. 


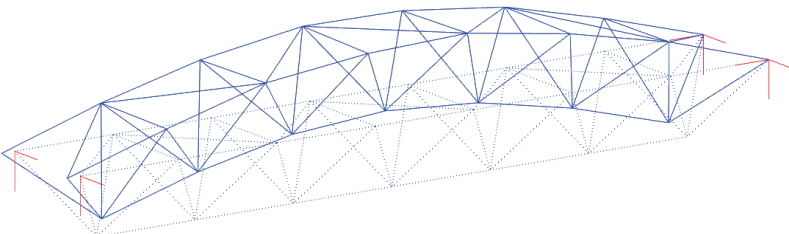

a) $1^{\text {st }}$ vertical mode, $\omega_{1}=368 \mathrm{rad} / \mathrm{s}$

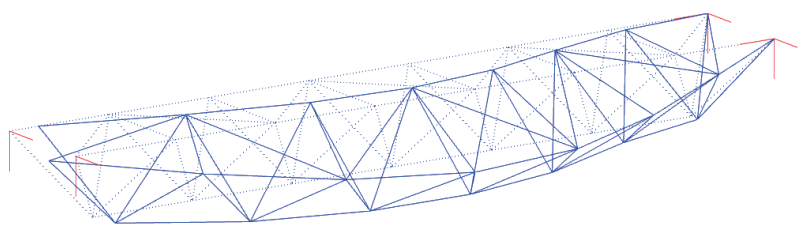

b) $1^{\text {st }}$ lateral mode, $\omega_{2}=604 \mathrm{rad} / \mathrm{s}$

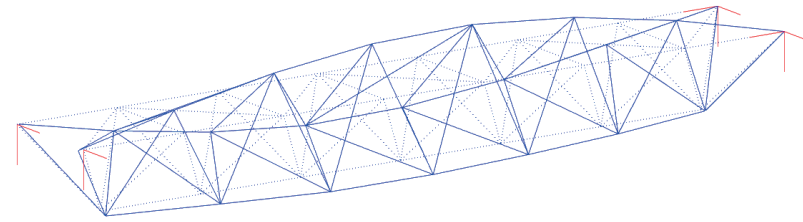

c) $1^{\text {st }}$ torsional mode, $\omega_{3}=950 \mathrm{rad} / \mathrm{s}$

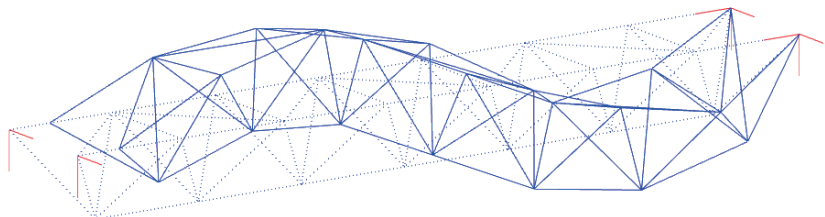

d) $2^{\text {nd }}$ vertical mode, $\omega_{4}=1100 \mathrm{rad} / \mathrm{s}$

Fig. 5 Mode shapes of MERO truss

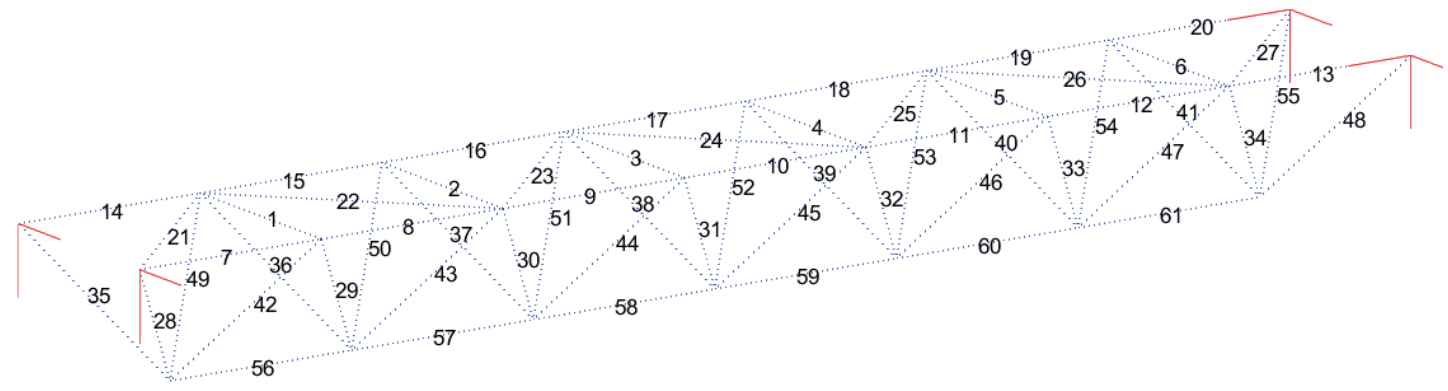

Fig. 6 Element numbering of the MERO truss

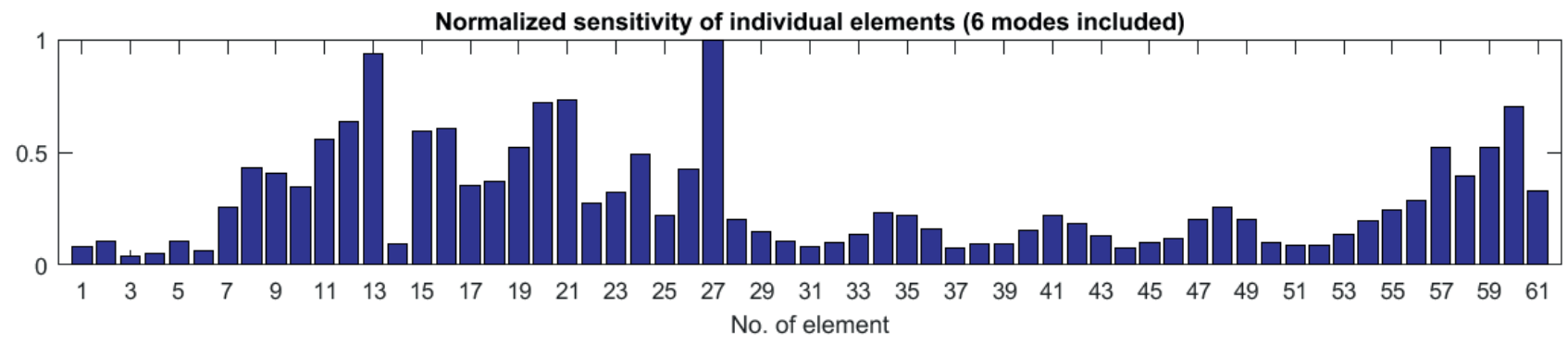

Fig. 7 Cumulative element sensitivity of the first six natural frequencies to stiffness modification

Beam elements of this kind of structure can be classified into one of the two groups depending on their function (Fig. 6):

- main elements, transferring loads to the supports,

- auxiliary elements.

Within the first group we can further distinguish upper (no. 7 to 20) and lower chord elements (no. 56 to 61). The second group consists of horizontal elements (no. 1 to 6 ) and diagonals (remaining elements).

The rationale behind the above classification can be better understood by looking at the cumulative sensitivity of the first six natural frequencies to stiffness modification in individual groups (Fig. 7). In this figure we can see that, except element no. 14 attached to the roller support, lower and upper chord elements have strong influence on the change in modal characteristics, while modification of stiffness in diagonals has a smaller effect on this change. The situation is even worse when we look at the horizontal elements which have marginal influence not only on the first or second, but all first six natural frequencies. This fact should be taken into account when designing an SHM system for this kind of structures.

The next step in the proposed methodology for damage identification is the selection of damage-sensitive locations for potential placement of sensors. For that purpose one needs to determine the sensitivity of individual degrees of freedom of the investigated structure. This can be done using the mode shape sensitivity matrix introduced in Section 2.2. Sensitivity analysis provides information presented in Fig. 9 and a node numbering helpful in sensor positioning is shown in Fig. 8. 


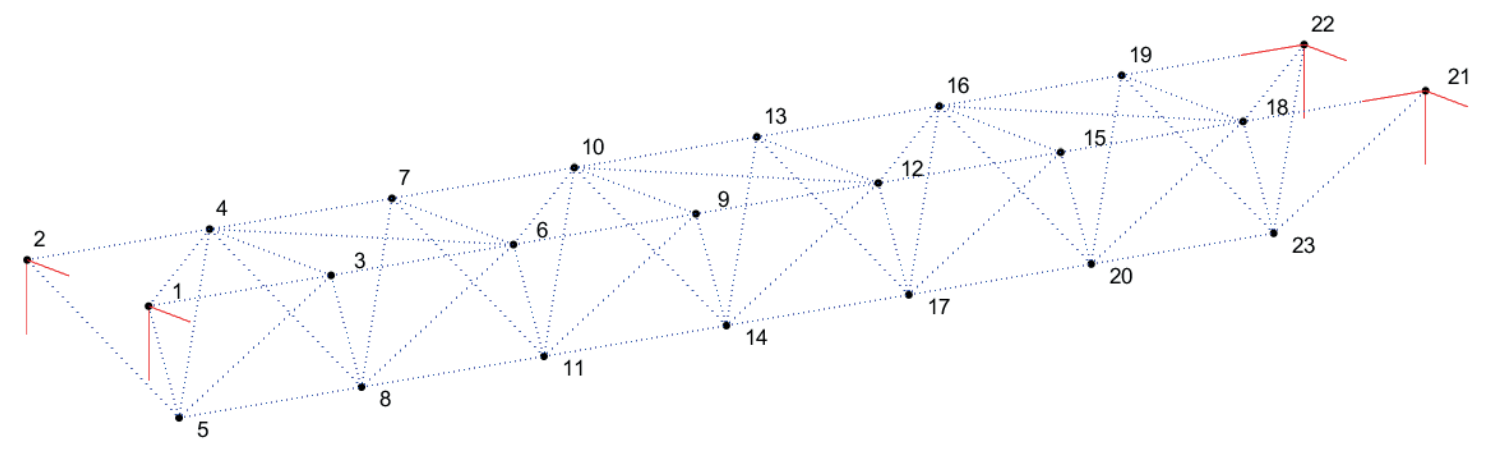

Fig. 8 Node numbering of the MERO truss

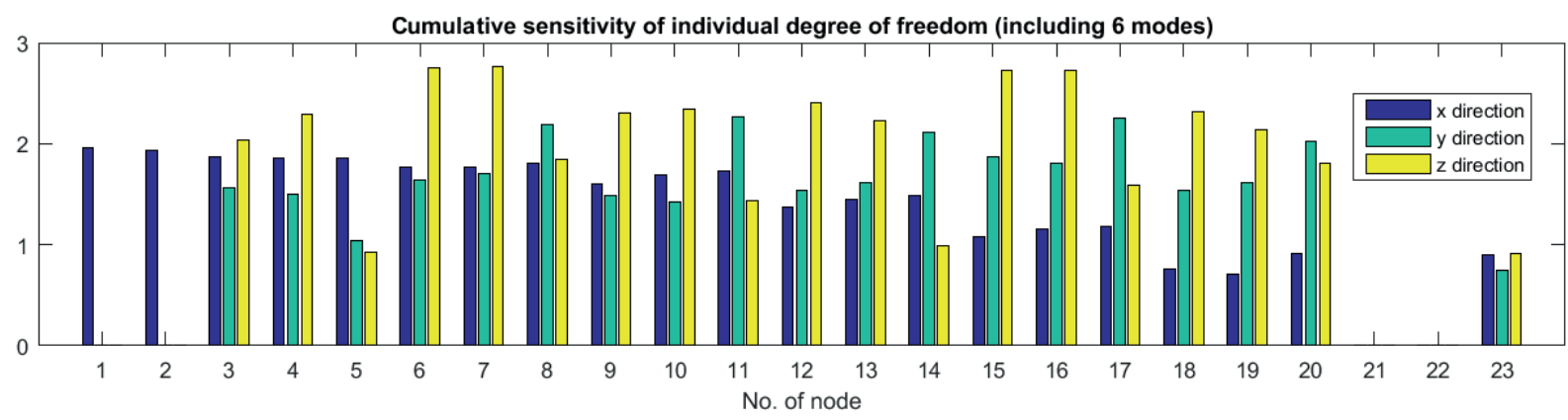

Fig. 9 Mode shape sensitivity at individual DOFs (6 modes taken into consideration)

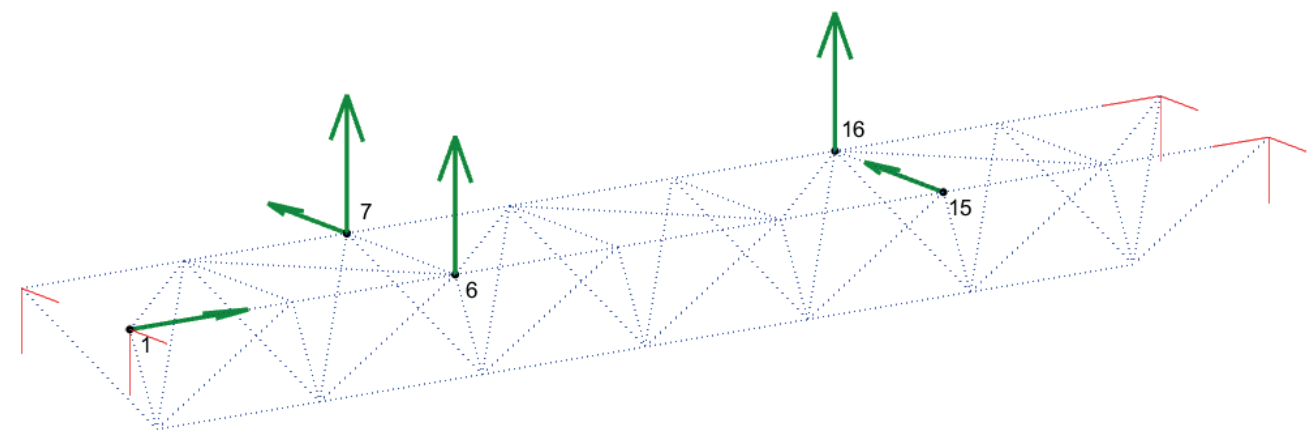

Fig. 10 Sensor placement using Effective Independence method

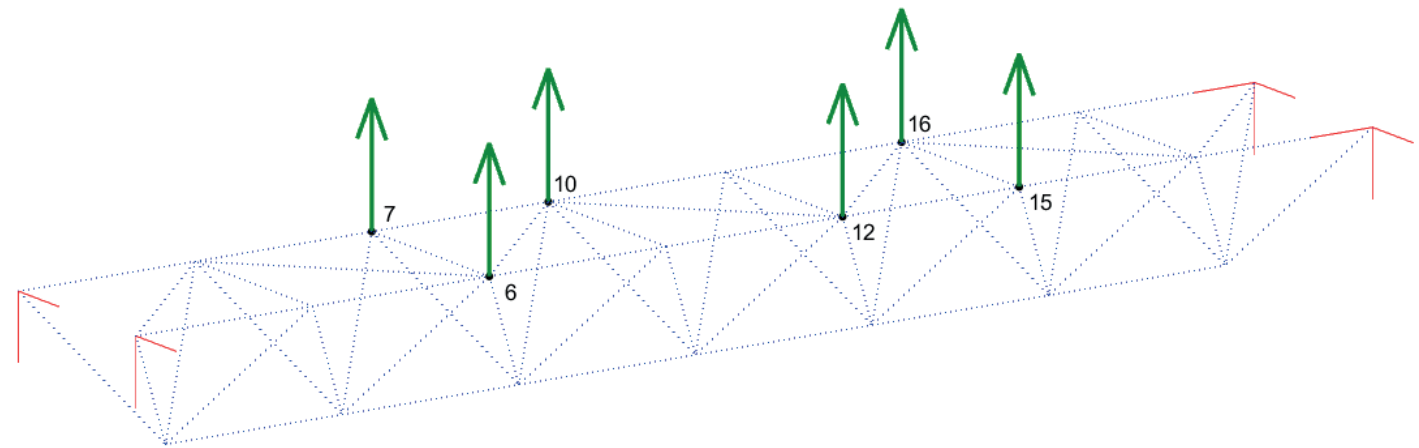

Fig. 11 Sensor placement using damage-sensitive method

Based on Fig. 9, the following conclusions can be drawn: the most sensitive locations are at nodes no. 6 with 7 and then 15 with 16 . This is manifested by the highest values of sensitivities in the vertical direction (yellow bars). For positioning of the next two sensors, the candidate location would be 12 and 10 .
For comparison of the damage-sensitive sensor placement methodology presented here with one of the most frequently used methods called Effective Independence, both strategies have been tested and the results are presented in Figs. 10 and 11. 


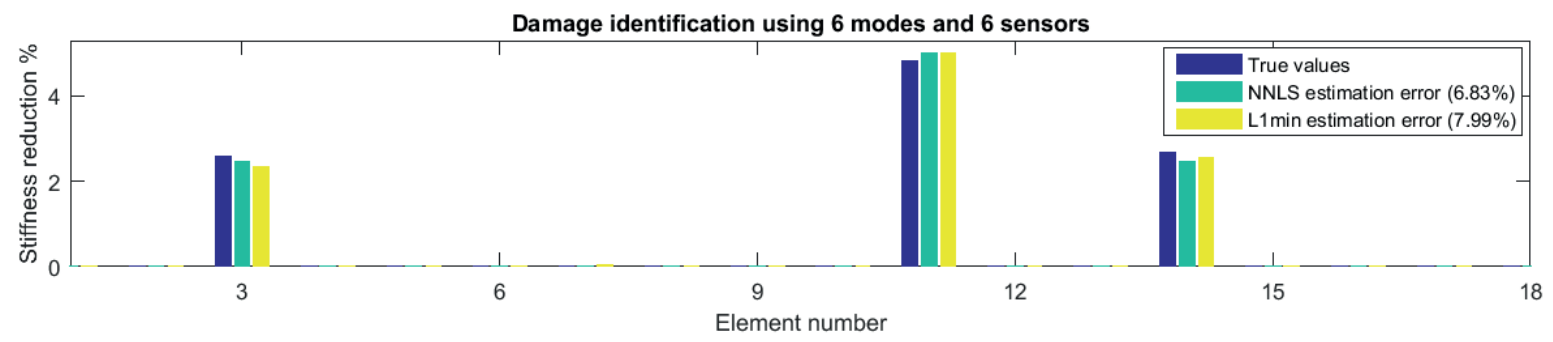

Fig. 12 Multiple damage identification in the MERO truss (6 modes with 6 sensors)

Table 2 Single damage element identifiability in 3D truss structure

\begin{tabular}{|c|c|c|c|c|c|c|c|c|c|c|c|}
\hline & \multicolumn{11}{|c|}{ No. of modes } \\
\hline \multirow{9}{*}{$\begin{array}{l}n \\
0 \\
0 \\
0 \\
0 \\
\infty \\
4 \\
0 \\
\dot{0} \\
z\end{array}$} & & & 1 & & 2 & & 3 & & 4 & & 5 \\
\hline & 1 & 6 & (4) & 31 & (8) & 38 & (17) & 55 & (23) & 59 & (35) \\
\hline & 2 & & (6) & 45 & (15) & 53 & (25) & 61 & (34) & 61 & (49) \\
\hline & 3 & 25 & (8) & 50 & (21) & 59 & (38) & 61 & (49) & 61 & (55) \\
\hline & 4 & 34 & (13) & 53 & (25) & 61 & (43) & 61 & (54) & 61 & (57) \\
\hline & 5 & 36 & (18) & 56 & (28) & 61 & (44) & 61 & (54) & 61 & (59) \\
\hline & 6 & 44 & (19) & 57 & (35) & 61 & (46) & 61 & (56) & 61 & (61) \\
\hline & 7 & 47 & (24) & 57 & (40) & 61 & (48) & 61 & (56) & 61 & (61) \\
\hline & 8 & 47 & (30) & 57 & (51) & 61 & (51) & 61 & (59) & 61 & (61) \\
\hline
\end{tabular}

In the analyzed MERO structure, both methods gave similar results as concerns selecting vertical directions in nodes 6,7 and 16 . The remaining sensor locations differ and highlight the difference between methods. While Effective Independence tries to maximize the determinant of the Fisher information matrix for mode shapes itself, the damage-sensitive method selects locations with the highest mode shape sensitivity metrics.

The third step in the proposed methodology is verification of the damage identifiability. For that purpose we assume the number of excited modes and number of sensors. Then, we are using a sparsity constrained optimization to identify damage in every structural member. The damage is introduced by reducing stiffness of an element by $1 \%$ of its original value. The effectiveness of the two algorithms described in Section 2.3, namely: $\ell_{1}$-norm minimization (L1 min) and non-negative least squares (NNLS), is compared.

The results are presented in Table 2. Values in parentheses correspond to the L1min method. Generally, NNLS demonstrates better performance in damage identification than L1min and the remaining analysis will refer to that algorithm. Additionally, we can observe that using only measurements from 1 sensor for a structure excited only with the first mode, both algorithms are able to identify correctly at most 6 damaged elements. Identification of all 61 elements is possible when at least three modes are excited and the structure is equipped with 4 sensors, or one excites 4 modes and uses more than 2 sensors.
Another important observation is related to the number of sensors versus number of excited modes. Increasing the number of excited modes results in better identifiability of the system parameters than increasing the number of sensors.

Finally, identification of multiple damage scenarios has been tested using both algorithms. A large number of randomly selected damage scenarios have been analyzed and again the NNLS algorithm gave a higher accuracy of estimation. One example of a multiple damage scenario with the applied modification and those identified is shown in Fig. 12. The values in parentheses in this figure represent estimation error.

\subsection{Influence of measurement noise and modelling errors}

Identification of damage scenario presented at the end of previous subsection has been performed under assumption that measured modal parameters are free of measurement noise and the mathematical model of structure under investigation is known accurately. In reality, however, measurements are often corrupted by noise and model of the structure contains some modelling errors. The source of these errors can be related to uncertain boundary conditions or unknown stiffness of semi-rigid connections. Taking into account the above aspects Eq. (13) should be modified to the following form

$\Delta \mathbf{b}=\mathbf{S} \Delta \boldsymbol{\theta}+\boldsymbol{\varepsilon}$,

where $\varepsilon$ represents errors caused by measurement noise and modeling simplifications.

The difference between these two type of errors is related to the fact that measurement noise is random and independent at every sensor location. In contrary modelling errors have systematic character and exhibit certain correlation.

To investigate the influence of the above errors on the damage identification process with aid of proposed methodology, the truss structure presented in previous subsection has been analyzed. The first identification has been 

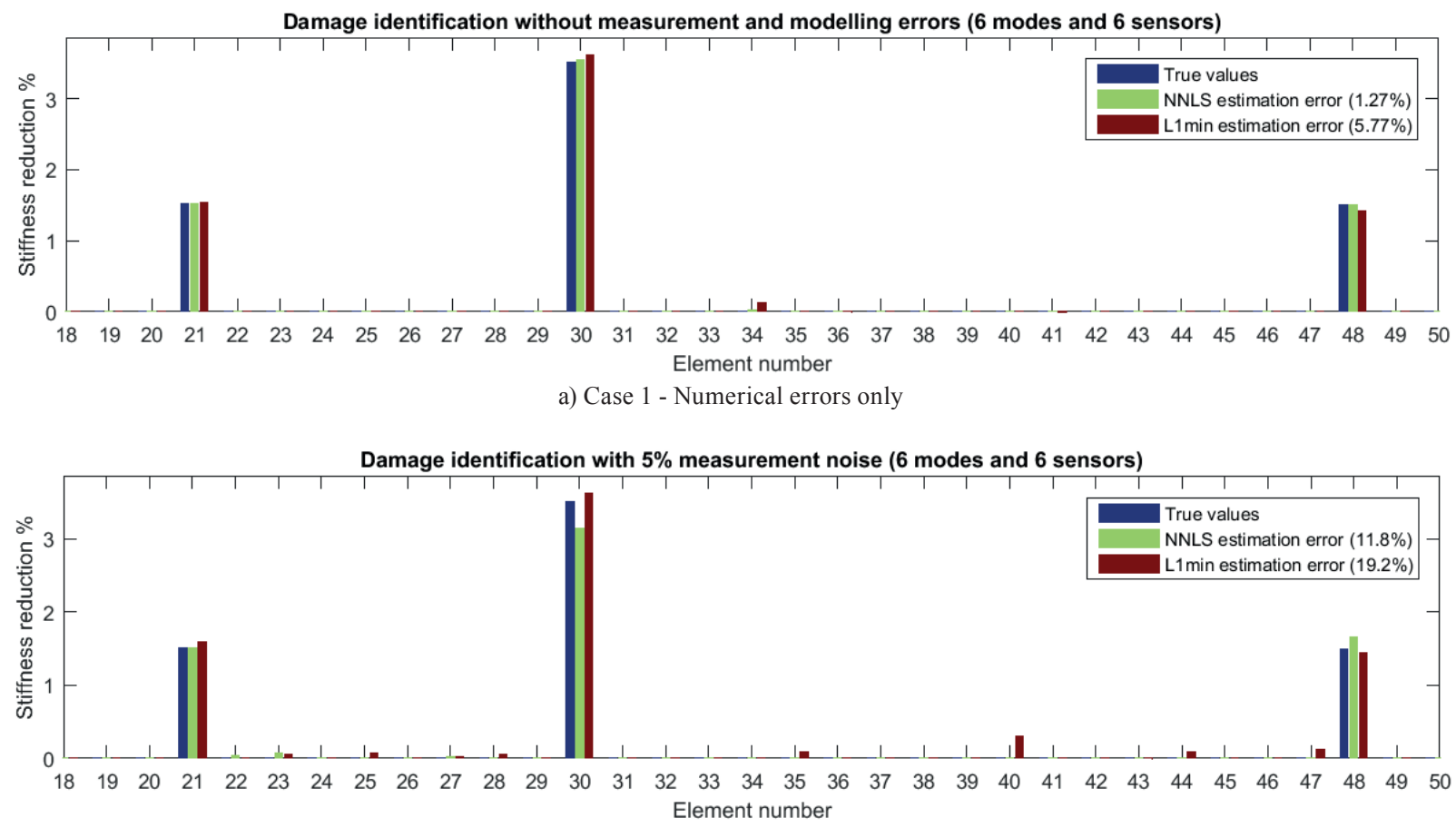

b) Case 2 - Numerical and measurement errors

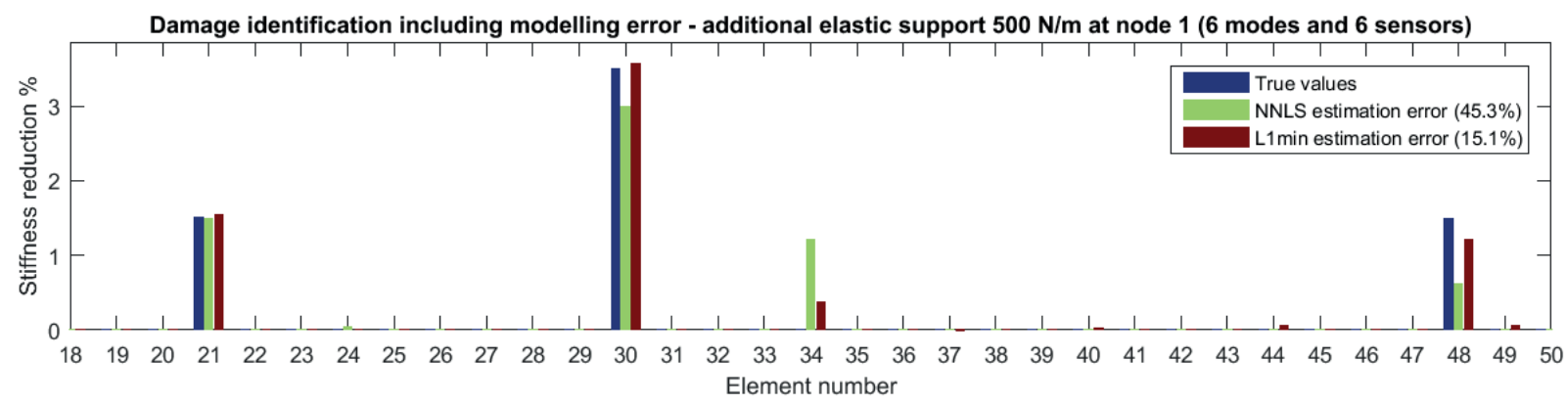

c) Case 3 - Numerical and modelling errors

Fig. 13 Influence of measurement noise and modelling errors on accuracy of damage identification

performed for ideal case which was free of measurement and modelling errors (Fig. 13a). The estimation errors presented in this figure are related to numerical accuracy of the applied algorithm only.

The next case takes into account the measurement errors (Fig. 13b). It was assumed that the measured natural frequencies and mode shapes are corrupted by $5 \%$ error. As can be observed in the figure NNLS and L1min algorithms still correctly localize damaged elements. However, the estimation errors are higher than in previous case and their values exceed $10 \%$.

The third analyzed case included modelling errors coming from unmodelled elastic support with stiffness equal to $500 \mathrm{~N} / \mathrm{m}$.

This additional support acts at node no. 1 in $\mathrm{x}$-direction and makes the structure stiffer than the nominal one. Similarly as in the case of measurement noise the identification procedure localize damaged elements properly (Fig. 13c). However, in this case false positive element no. 34 is reported by both algorithms.

The comparison of these three cases allows to conclude that the proposed methodology for sparsity constrained damage identification is relatively insensitive to measurement and modelling errors and should be suitable for real engineering applications.

\subsection{Steel arch bridge - $\mathbf{4 1 6}$ member structure}

The second case study consists of a full-scale arch bridge composed of 416 steel members. The material and geometrical data for this structure have been taken from the paper by Lu et al. [28]. While in this paper seismic performance of the bridge is evaluated, here we consider the required number of sensors and modes in order to assure full identifiability of this structure. 


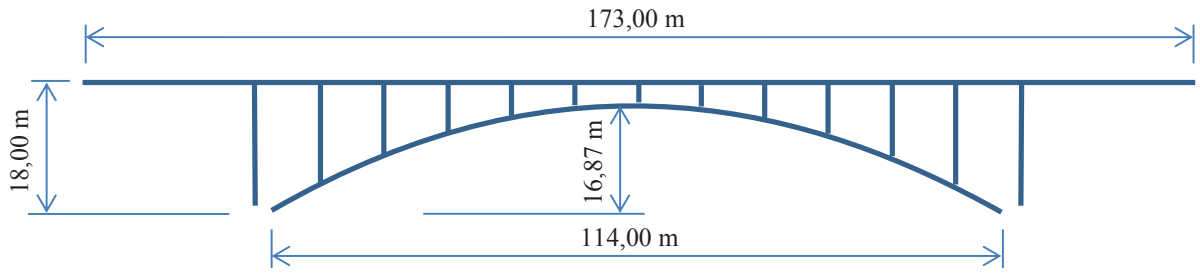

Fig. 14 Elevation of the investigated steel arch bridge

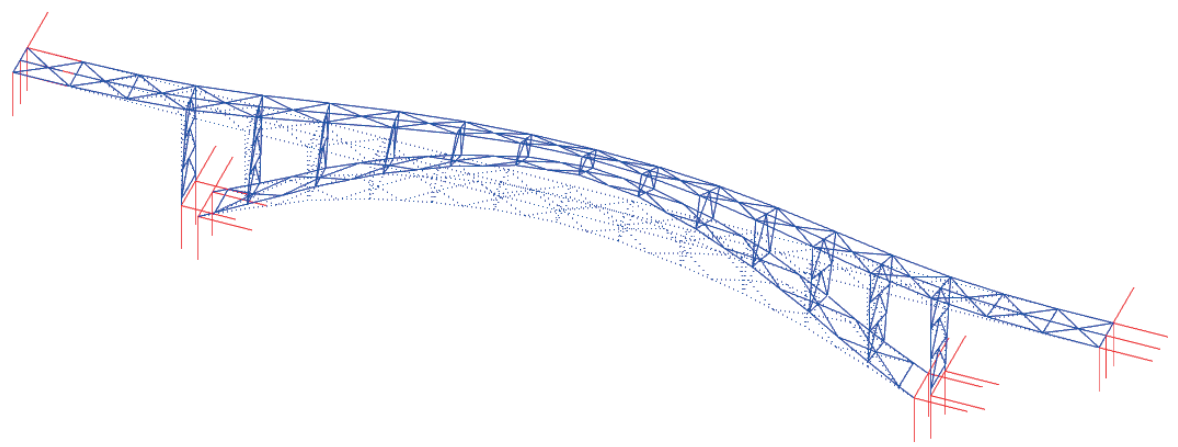

(a) $1^{\text {st }}$ lateral mode, $\omega_{1}=10.6 \mathrm{rad} / \mathrm{s}$

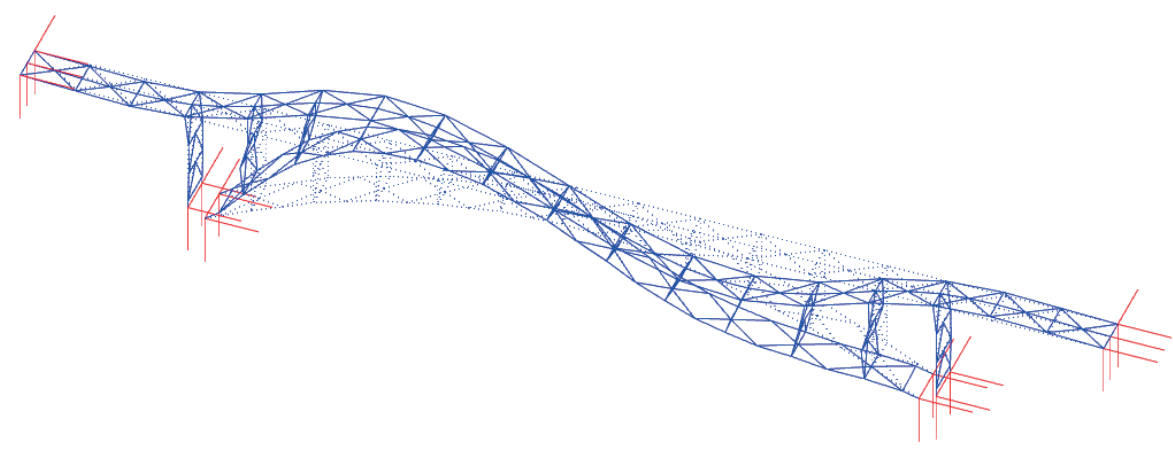

(b) $1^{\text {st }}$ vertical mode, $\omega_{2}=13.9 \mathrm{rad} / \mathrm{s}$

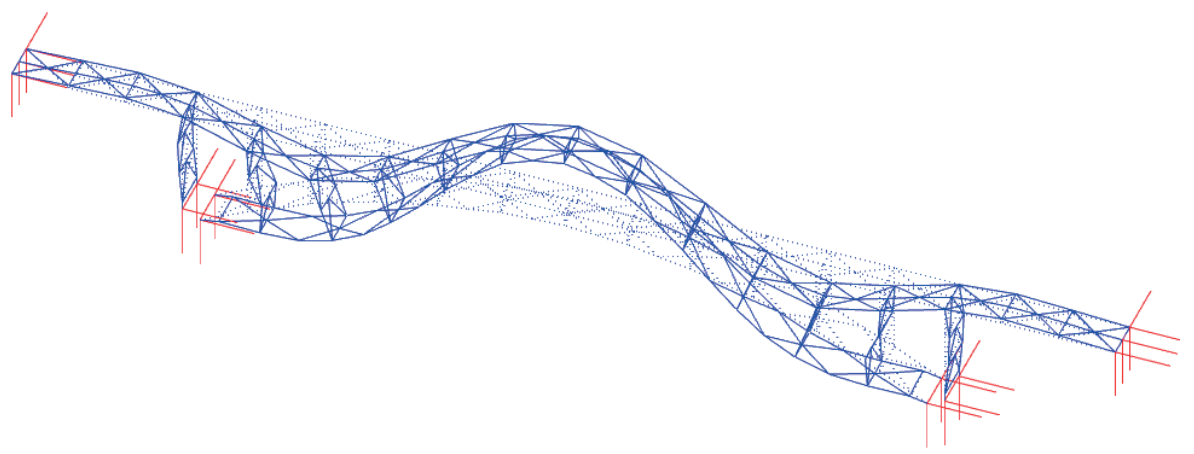

(c) $2^{\text {nd }}$ vertical mode, $\omega_{3}=17.9 \mathrm{rad} / \mathrm{s}$

Fig. 15 First three mode shapes and natural frequencies of the arch bridge

The first three mode shapes of the structure are presented in Fig. 15.

The first step of the damage identification, similarly as in the case of the MERO truss, was the calculation of modal sensitivities for both parametric sensitivity verification and sensor placement. The first sixteen modal frequencies and corresponding mode shapes have been used in that sensitivity analysis. Then, again Effective Independence based sensor placement has been compared with that obtained by the damage-sensitivity method. As in the case of the MERO truss, sensor distributions obtained using both methods are quantitatively quite similar with the preferred sensor location on the steel arch in the vertical and horizontal directions. 
Table 3 Single damage element identifiability in arch bridges

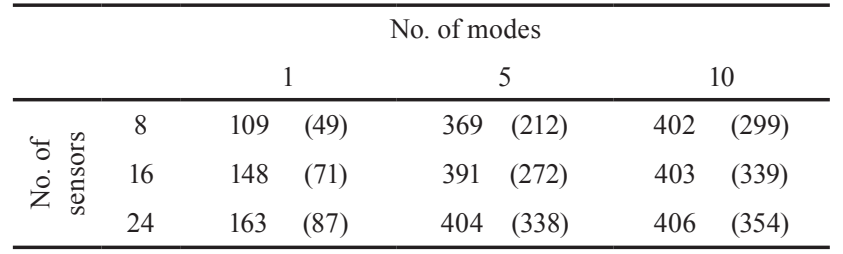

The results of the second step of the proposed methodology, which is identification of a single damaged element, are presented in Table 2. Similarly as in the case of the MERO truss, better results have been obtained from the NNLS algorithm. Using 16 sensors and exciting 10 modes it was possible to identify correctly a single damage in 406 out of 416 structural members. The remaining 10 elements were hardly identifiable even using a larger number of sensors and modes. However, investigation of the results revealed that these were the elements connected to supports and to assure their proper identification they should be possibly equipped with dedicated monitoring systems.

\section{Conclusions}

A comprehensive methodology for damage localization and quantification has been proposed. It consists of three levels of investigation. Firstly, it utilizes sensitivity analysis to determine the influence of stiffness modification of individual structural members on changes in modal characteristics of the structure. The second level of the proposed methodology uses the sensitivity matrix of the natural frequencies to determine hardly identifiable parameters. Simultaneously, sensitivity of the corresponding mode shapes helps to select damage-sensitive positions of sensors. The third level applies two sparsity constrained optimization algorithms to efficiently identify the applied damage scenarios. These two algorithms are based on $\ell_{1}$-norm minimization and non-negative least square (NNLS) solution, respectively. In both presented case studies of a three-dimensional truss girder and a steel arch bridge better results have been achieved using the NNLS algorithm. Moreover, the performed analyses

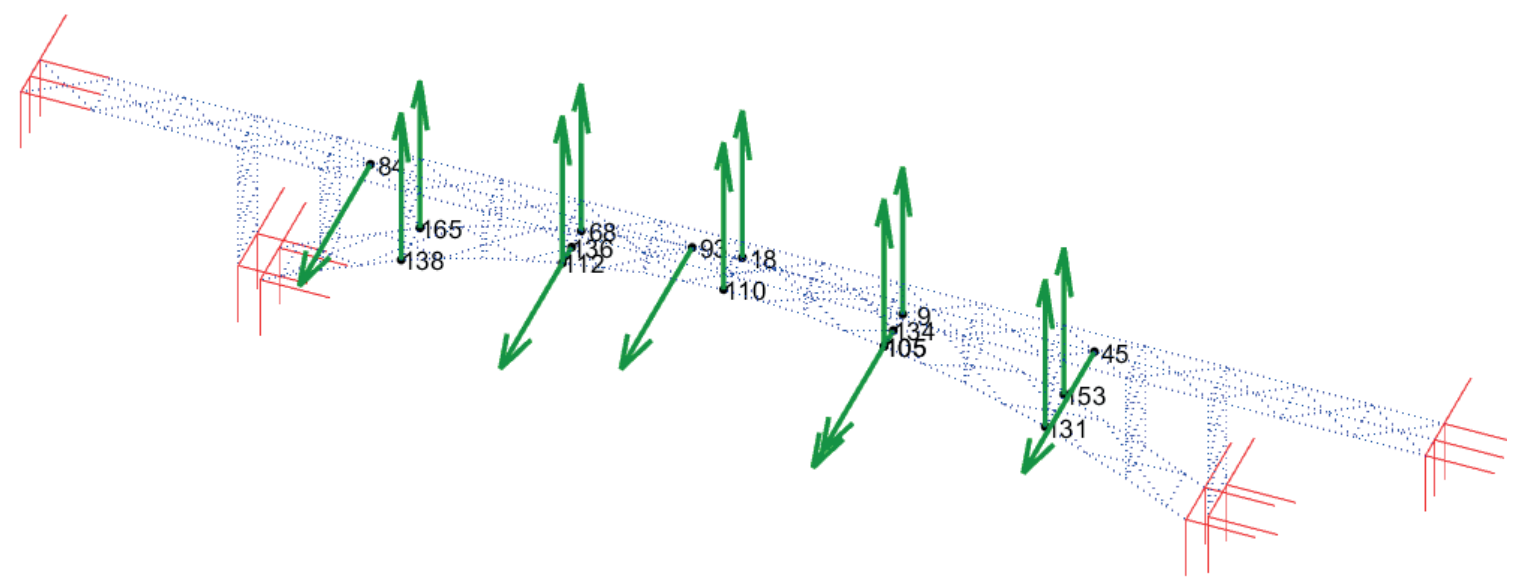

Fig. 16 Sensor placement on the arch bridge using Effective Independence method

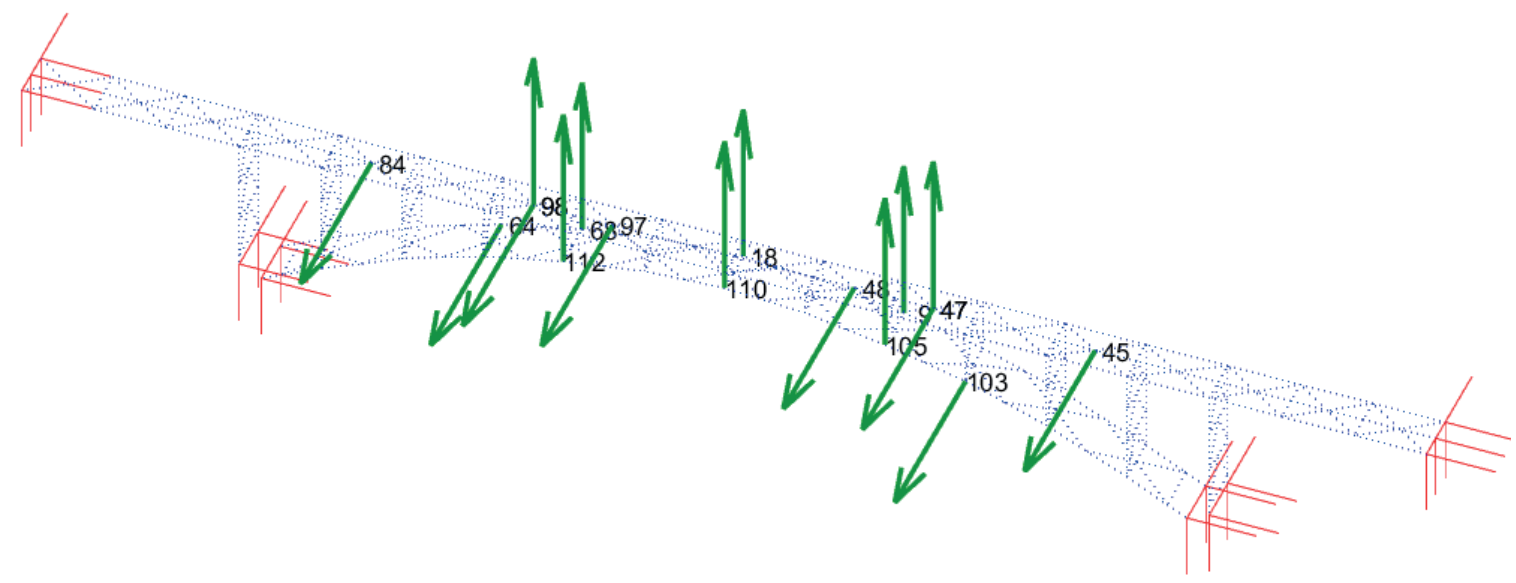

Fig. 17 Sensor placement on the arch bridge using damage-sensitive method 
revealed that excitation of more modes is more advantageous than measurement of more degrees of freedom. This observation can be useful in designing and implementing the SHM system in real complex engineering structures.

Finally, robustness of the proposed methodology in the presence of measurement and modelling errors has been demonstrated.

\section{Acknowledgement}

The support of the National Science Centre, Poland, granted under grant agreement DEC2017/25/B/ST8/01800, is gratefully acknowledged.

\section{Abbreviations}

The following abbreviations are used in this manuscript:

DoF Degree of freedom

EI Effective Independence

EKF Extended Kalman Filter

FEM Finite element method

FIM Fisher information matrix

IE Information entropy

MAC Modal assurance criterion

NNLS Non-negative least squares

OSP Optimal sensor placement

SHM Structural Health Monitoring

\section{References}

[1] Shah, P. C., Udwadia, F. E. "A Methodology for Optimal Sensor Locations for Identification of Dynamic Systems", Journal of Applied Mechanics, 45(1), pp. 188-196, 1978. https://doi.org/10.1115/1.3424225

[2] Cawley, P., Adams, R. D. "The location of defects in structures from measurements of natural frequencies", The Journal of Strain Analysis for Engineering Design, 14(2), pp. 49-57, 1979. https://doi.org/10.1243/03093247V142049

[3] Kammer, D. C. "Sensor placement for on-orbit modal identification and correlation of large space structures", Journal of Guidance, Control, and Dynamics, 14(2), pp. 251-259, 1991. https://doi.org/10.2514/3.20635

[4] Liu, P.-L., Chian, C.-C. "Parametric Identification of Truss Structures Using Static Strains", Journal of Structural Engineering, 123(7), pp. 927-933, 1997.

https://doi.org/10.1061/(ASCE)0733-9445(1997)123:7(927)

[5] Cobb, R. G., Liebst, B. S. "Sensor Placement and Structural Damage Identification from Minimal Sensor Information", AIAA Journal, 35(2), pp. 369-374, 1997. https://doi.org/10.2514/2.103

[6] Katafygiotis, L. S., Beck, J. L. "Updating Models and Their Uncertainties. II: Model Identifiability", Journal of Engineering Mechanics, 124(4), pp. 463-467, 1998.

https://doi.org/10.1061/(ASCE)0733-9399(1998)124:4(463)
[7] Papadimitriou, C. "Optimal sensor placement methodology for parametric identification of structural systems", Journal of Sound and Vibration, 278(4-5), pp. 923-947, 2004.

https://doi.org/10.1016/j.jsv.2003.10.063

[8] Barthorpe, R. J., Worden, K. "Sensor Placement Optimization". In: Boller, C., Chang, F.-K., Fujino, Y. (eds.) Encyclopedia of Structural Health Monitoring, 1st ed., John Wiley \& Sons, Hoboken, New Jersey, United States, 2009. https://doi.org/10.1002/9780470061626.shm086

[9] Zhou, K., Wu, Z. Y., Yi, X. H., Zhu, D. P., Narayan, R., Zhao, J. "Generic Framework of Sensor Placement Optimization for Structural Health Modeling", Journal of Computing in Civil Engineering, 31(4), 2017.

https://doi.org/10.1061/(ASCE)CP.1943-5487.0000662

[10] Friswell, M., Mottershead, J. "Finite Element Model Updating in Structural Dynamics", 1st ed., Springer Nature, Dordrecht, Netherlands, 1995. https://doi.org/10.1007/978-94-015-8508-8

[11] Hernandez, E. M. "Identification of isolated structural damage from incomplete spectrum information using 11-norm minimization", Mechanical Systems and Signal Processing, 46(1), pp. 59-69, 2014. https://doi.org/10.1016/j.ymssp.2013.12.009

[12] Ebrahimian, H., Astroza, R., Conte, J. P. "Extended Kalman filter for material parameter estimation in nonlinear structural finite element models using direct differentiation method", Earthquake Engineering \& Structural Dynamics, 44(10), pp. 1495-1522, 2015. https://doi.org/10.1002/eqe. 2532

[13] Ginsberg, D., Fritzen, C.-P., Loffeld, O. "Sparsity-constrained Extended Kalman Filter concept for damage localization and identification in mechanical structures", Smart Structures and Systems, 21(6), pp. 741-749, 2018.

https://doi.org/10.12989/sss.2018.21.6.741

[14] An, Y., Blachowski, B., Ou, J. "A degree of dispersion-based damage localization method", Structural Control and Health Monitoring, 23(1), pp. 176-192, 2016.

https://doi.org/10.1002/stc.1760

[15] Pnevmatikos, N. G., Blachowski, B., Hatzigeorgiou, G. D., Swiercz, A. "Wavelet analysis based damage localization in steel frames with bolted connections", Smart Structures and Systems, 18(6), pp. 1189-1202, 2016. https://doi.org/10.12989/sss.2016.18.6.1189

[16] Blachowski, B., An, Y., Spencer Jr., B. F., Ou, J. "Axial Strain Accelerations Approach for Damage Localization in Statically Determinate Truss Structures", Computer-Aided Civil and Infrastructure Engineering, 32(4), pp. 304-318, 2017. https://doi.org/10.1111/mice.12258

[17] An, Y., Blachowski, B., Zhong, Y., Holobut, P., Ou, J. "Rankrevealing $\mathrm{QR}$ decomposition applied to damage localization in truss structures", Structural Control and Health Monitoring, 24(2), 2017. https://doi.org/10.1002/stc.1849

[18] Blachowski, B., Swiercz, A., Gutkiewicz, P., Szelazek, J., Gutkowski, W. "Structural damage detectability using modal and ultrasonic approaches", Measurement, 85, pp. 210-221, 2016. https://doi.org/10.1016/j.measurement.2016.02.033 
[19] Blachowski, B., Gutkowski, W. "Effect of damaged circular flangebolted connections on behaviour of tall towers, modelled by multilevel substructuring", Engineering Structures, 111, pp. 93-103, 2016. https://doi.org/10.1016/j.engstruct.2015.12.018

[20] Blachowski, B., Swiercz, A., Jankowski, L. "Virtual Distortion Method based optimal sensor placement for damage identification", presented at ISMA 2018 / USD 2018, International Conference on Noise and Vibration Engineering / International Conference on Uncertainty in Structural Dynamics, Leuven, Belgium, Sept. 17-19, 2018.

[21] Kourehli, S. S. "Damage Diagnosis of Structures Using Modal Data and Static Response", Periodica Polytechnica Civil Engineering, 61(1), pp. 135-145, 2017. https://doi.org/10.3311/PPci.7646

[22] Bayer, J., Král, J., Urushadze, S. "Localization of Simulated Damage on a Steel Beam from Random Vibrations", Periodica Polytechnica Civil Engineering, 62(1), pp. 112-116, 2018. https://doi.org/10.3311/PPci.10625

[23] Bagherahmadi, S. A., Seyedpoor, S. M. "Structural damage detection using a damage probability index based on frequency response function and strain energy concept", Structural Engineering and Mechanics, 67(4), pp. 327-336, 2018.

https://doi.org/10.12989/sem.2018.67.4.327
[24] Beben, D. "Application of Interferometry Method for Dynamic Continuous Testing of Bridges", Periodica Polytechnica Civil Engineering, 60(3), pp. 387-395, 2016. https://doi.org/10.3311/PPci.8679

[25] Nelson, R. B. "Simplified calculation of eigenvector derivatives", AIAA Journal, 14(9), pp. 1201-1205, 1976. https://doi.org/10.2514/3.7211

[26] Hou, R., Xia, Y., Bao, Y., Zhou, X. "Selection of regularization parameter for 11-regularized damage detection", Journal of Sound and Vibration, 423, pp. 141-160, 2018. https://doi.org/10.1016/j.jsv.2018.02.064

[27] Suwała, G., Jankowski, Ł. "Nonparametric identification of structural modifications in Laplace domain", Mechanical Systems and Signal Processing, 85, pp. 867-878, 2017. https://doi.org/10.1016/j.ymssp.2016.09.018

[28] Lu, Z., Ge, H., Usami, T. "Applicability of pushover analysis-based seismic performance evaluation procedure for steel arch bridges", Engineering Structures, 26(13), pp. 1957-1977, 2004. https://doi.org/10.1016/j.engstruct.2004.07.013 